\title{
Wind Turbine Radar Cross Section
}

\author{
David Jenn ${ }^{1}$ and Cuong Ton ${ }^{2}$ \\ ${ }^{1}$ Naval Postgraduate School, 833 Dyer Road, Room 437, Monterey, CA 93943, USA \\ ${ }^{2}$ Naval Air Warfare Center, Weapons Division, Point Mugu, CA 93042, USA \\ Correspondence should be addressed to David Jenn, jenn@nps.edu
}

Received 12 October 2012; Accepted 4 December 2012

Academic Editor: James Matthews

Copyright $\odot 2012$ D. Jenn and C. Ton. This is an open access article distributed under the Creative Commons Attribution License, which permits unrestricted use, distribution, and reproduction in any medium, provided the original work is properly cited.

The radar cross section (RCS) of a wind turbine is a figure of merit for assessing its effect on the performance of electronic systems. In this paper, the fundamental equations for estimating the wind turbine clutter signal in radar and communication systems are presented. Methods of RCS prediction are summarized, citing their advantages and disadvantages. Bistatic and monostatic RCS patterns for two wind turbine configurations, a horizontal axis three-blade design and a vertical axis helical design, are shown. The unique electromagnetic scattering features, the effect of materials, and methods of mitigating wind turbine clutter are also discussed.

\section{Introduction}

Wind power installations (wind farms) are increasing globally at a rate of about 20 percent annually $[1,2]$. The increasing number and density of wind farms is putting them into closer proximity of microwave transmission and reception facilities such as radar, radio, television, GPS, cellular, and wireless networks. The receivers in these systems rely on detecting and processing very weak signals. Wind farms, and even individual wind turbines, can significantly affect the received signals in many cases. The large scattering cross sections of the towers and blades result in strong signals that can saturate the receiver or mask the desired signals. Furthermore, the motion of the blades introduces a Doppler shift that can degrade the processing gain. To assure that a high-performance sensor or communication system can operate in the vicinity of wind farms, detailed analysis, measurements, or simulations may have to be conducted.

The radar cross section (RCS) is a figure of merit that can serve to estimate the effect of a wind turbine on a system's performance. Numerous studies have been performed evaluating the RCS of wind farms and their effect on radar and communication systems. Studies on wind turbine impact on radar performance appear in [3-8]. References $[3,6,8]$ have used measurements, either in the field or a measurement facility, to estimate the wind turbine scattering and its impact on radar performance. Reference
[9] discusses the more general problem of RF installations with wind turbines in the near field of the antenna, and [10] specifically addresses the interference with digital television reception. References [11-13] address the reduction and control of wind turbine RCS, in order to minimize the interference with RF systems.

In this paper, some fundamental scattering characteristics of wind turbine RCS are examined. In Section 2, the RCS is defined and the basic equations for predicting the scattered power from a wind turbine are presented. Section 3 discusses the various computational methods that can be used to predict RCS, citing their advantages and disadvantages. Section 4 shows RCS simulation results for two wind turbine configurations: a horizontal axis threeblade design and a vertical axis helical design. Section 5 addresses the issue of RCS reduction and control for wind turbines. Finally, Section 6 contains a summary of the results, conclusions, and suggested directions for future research.

\section{Radar Range and Link Equations}

Figure 1 shows a general scenario with multiple electronic communication and sensor systems operating in the vicinity of wind farms. The majority of current operational radar systems are monostatic, where the transmitter and receiver are colocated as viewed from the target. Radar returns from 


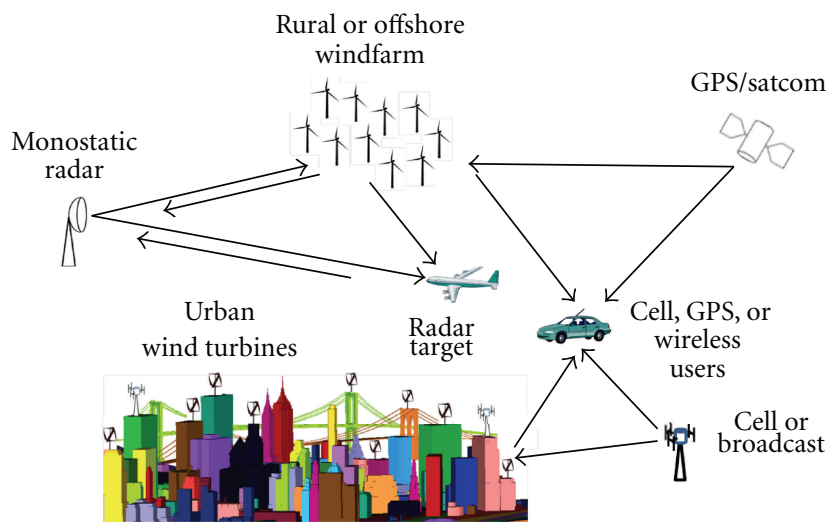

Figure 1: Monostatic and bistatic geometries encountered by electronic communication and sensor systems.

a wind farm can be much larger than most targets, making the detection and tracking of objects traversing the wind farm difficult [3]. Even if the target is outside of the wind farm area, strong returns from the tower and blades can mask weak target returns. The rotor-induced Doppler spread can mask moving targets or be mistaken for weather echoes. The wind turbine portion of the return, due to its characteristics, may not be recognized and processed as clutter by the radar.

The systems under consideration are sufficiently narrowband so that phasor notation can be used ( $e^{j \omega t}$ time dependence assumed and suppressed) and analysis performed at a single frequency, typically the carrier frequency.

The RCS of a point target (i.e., a target whose extent is much less than the size of the radar resolution cell) is defined for a plane wave incident $[14,15]$

$$
\sigma_{p q}\left(f, \theta_{i}, \phi_{i}, \theta_{s}, \phi_{s}\right)=\lim _{R \rightarrow \infty} \frac{4 \pi R^{2}\left|E_{p}^{s}\left(\theta_{s}, \phi_{s}\right)\right|^{2}}{\left|E_{q}^{i}\right|^{2}},
$$

where $R$ is distance from the target, $f$ is the frequency, the subscript/superscript $i$ denotes incident, $s$ scattered, and $p, q=\theta$ or $\phi$ are the components in a spherical polar coordinate system, as shown in Figure 2. The limiting process in (1) assures that the scattered field is proportional to $1 / R$.

The copolarized RCS refers to the case where $p=$ $q$, whereas the cross-polarized RCS is $p \neq q$. Generally, $\sigma$ is written as a scalar and the functional dependencies on frequency and angle are suppressed. The unit is typically $\mathrm{m}^{2}$ or the decibel unit $\mathrm{dBsm}$ defined by

$$
\sigma, \mathrm{dBsm}=10 \log _{10}\left(\sigma, \mathrm{m}^{2}\right) .
$$

The received power from a target at range $R$ for a monostatic radar is given by the conventional radar range equation (RRE) [16]

$$
S=\frac{P_{t} G_{t}^{2} \lambda^{2} \sigma_{t}}{(4 \pi)^{3} R^{4} L}\left|F_{t}\right|^{4}
$$

where $P_{t}$ is the transmitter power, $G_{t}$ the antenna gain in the direction of the target, $\lambda$ the wavelength, $\sigma_{t}$ the target RCS $\left(\mathrm{m}^{2}\right)$, and $L$ is a miscellaneous system loss factor.
The factor $F_{t}$ is the one-way voltage (or field) path gain (propagation) factor. It is squared to obtain power and squared again for round trip, resulting in a fourth power. The path gain factor is a complex quantity that accounts for the relevant propagation modes between the radar and target. Generally, since most of the systems under consideration operate near the ground, it would include multipath (or "ground bounce"). It would also include losses due to precipitation and foliage.

Normally radar and communication system performance measures such as probability of detection and probability of bit error are based on the signal-to-noise ratio (SNR). In our case, we will neglect the effects of noise in comparison to the wind turbine clutter and use the signal-to-clutter ratio (SCR) as the basis for performance evaluation. The clutter power return from a wind turbine point target with RCS $\sigma_{w}$ at the range $R_{w}$ is

$$
C=\frac{P_{t} G_{w}^{2} \lambda^{2} \sigma_{w}}{(4 \pi)^{3} R_{w}^{4} L}\left|F_{w}\right|^{4}
$$

Note that both the target and wind turbine RCSs are changing with time. For the wind turbine it is due to rotor motion and the associated change in multipath due to rotor motion; for the target it is due to changing aspect angle, velocity, and multipath.

From (3) and (4), we obtain the SCR for the monostatic case

$$
\frac{S}{C}=\left(\frac{G_{t}^{2}}{G_{w}^{2}}\right)\left(\frac{\sigma_{t}}{\sigma_{w}}\right)\left(\frac{R_{w}^{4}}{R^{4}}\right)\left(\frac{\left|F_{t}\right|^{4}}{\left|F_{w}\right|^{4}}\right) .
$$

It is seen that the SCR cannot be increased by increasing the transmitter power, because the clutter power increases along with the target power. Figure 3 illustrates the tradeoffs between the factors in (5) for a line-of-sight (LOS) condition $\left(\left|F_{t}\right|=\left|F_{w}\right| \approx 1\right)$. The curves are drawn for a reference of $\mathrm{SCR}_{\text {ref }}=0 \mathrm{~dB}$. The curves apply to any other SCR by moving them on the vertical axis by the difference SCR - SCR ref For the situation where both the target and wind turbine are not in the main beam, then low sidelobes are effective in reducing the interference. 


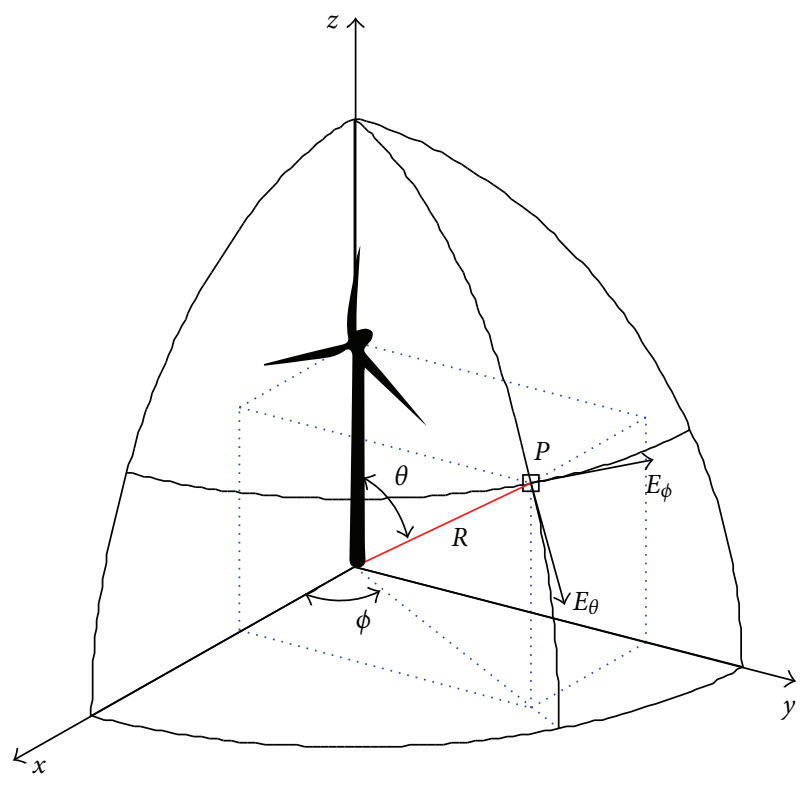

FIGURE 2: Coordinate system for RCS patterns.

Due to the complex propagation environment and geometry, the relative phases between the target and clutter components can be regarded as random. The total power in the receiver is determined using the sum of the complex voltages $V_{t}$ and $V_{w}$ due to the target and clutter returns, respectively. In this case, since we are interested in the average power, it is possible to approximate the total power received by the sum of the target and clutter powers. If the receiver impedance is $Z$ (real), then the total average received power is the noncoherent sum

$$
\begin{aligned}
P_{r}= & \left\langle\frac{\left(V_{t}+V_{w}\right)\left(V_{t}+V_{w}\right)^{*}}{Z}\right\rangle=\frac{\left|V_{t}+V_{w}\right|^{2}}{Z} \approx \frac{\left|V_{t}\right|^{2}}{Z} \\
& +\frac{\left|V_{w}\right|^{2}}{Z}=S+C,
\end{aligned}
$$

where \langle\rangle denotes expected value. This approximation allows us to treat each component individually.

Bistatic geometries occur when the transmitter and receiver are sufficiently separated in angle. Bistatic radar is not as common as monostatic radar; however, the general bistatic case would encompass broadcast systems, cellular radio, and GPS. Referring to Figure 4, the direct signal from the transmitter to the receiver is [17]

$$
S=\frac{P_{t} G_{t} G_{r} \lambda^{2}}{(4 \pi R)^{2} L}\left|F_{d}\right|^{2}
$$

where $R$ is the direct path distance between the transmitter and receiver, $G_{t}$ the transmit antenna gain in the direction of the receiver, $G_{r}$ the receive antenna gain in the direction of the transmitter, and $F_{d}$ is the one-way voltage (or field) direct path propagation factor.

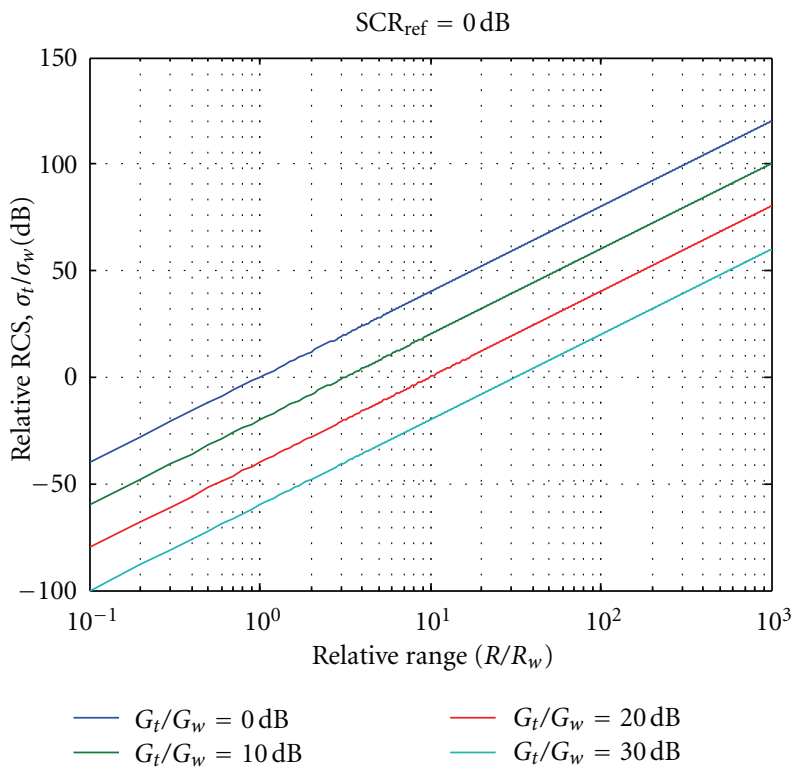

FIGURE 3: Universal curves illustrating the relationship between the factors in (5).

Again, referring to the bistatic geometry depicted in Figure 4 , the clutter power from the wind turbine arriving at the receiver is [18]

$$
C=\frac{P_{t} G_{t w} G_{r w} \lambda^{2} \sigma_{b w}}{(4 \pi)^{3} R_{t}^{2} R_{r}^{2} L_{t} L_{r}}\left|F_{t}\right|^{2}\left|F_{r}\right|^{2},
$$

where the subscript $w$ is used to denote wind turbine parameters. The subscript $t$ refers to transmit and $r$ to receive; $\sigma_{b w}$ is the wind turbine's bistatic RCS when the incidence direction is from the transmitter and the observation direction is from the receiver, as defined in Figure 4. 
The SCR for the special case of line-of-sight propagation path and no losses $\left(L_{t}, L_{r}, L,\left|F_{t}\right|,\left|F_{r}\right| \approx 1\right)$ gives

$$
\frac{S}{C}=\frac{G_{t} G_{r}}{G_{t w} G_{r w}} \frac{R_{t}^{2} R_{r}^{2}}{R^{2}} \frac{4 \pi}{\sigma_{b w}} .
$$

In order to increase the SCR, aside from reducing the wind turbine RCS, the sidelobe levels of the two antennas should be as low as possible.

\section{RCS Prediction Methods}

The determination of the RCS of complex objects, such as wind turbines, can be computationally demanding. It requires the numerical solution of some variation of Maxwell's equations, or high frequency approximations thereof, in either integral or differential form. Maxwell's equations are solved subject to the pertinent boundary conditions of the problem. Rigorous methods include the method of moments (MoM) solution of integral equations in the frequency domain [19], or the finite difference time domain (FDTD) solution of the differential equations in the time domain [20]. The finite element method (FEM) is also used in both the time and frequency domains [21]. Fourier transform relationships exist between the time and frequency domain solutions.

The MoM is appealing because it is a rigorous solution that includes all the interactions between currents on the structure, and thus all scattering mechanisms (multiple reflections, diffraction, surface waves, etc.). MoM requires surface meshing of the object into facets with edge lengths small compared to the wavelength. A matrix equation, of an order approximately equal to the number of internal edges, must be solved for the current. Then the current is used in the radiation integrals to obtain $E_{p}^{s}$ for use in (1). At high frequencies (e.g., $10 \mathrm{GHz}$ ), millions of unknowns may be required for a converged current series. This has prevented the wide-spread use of MoM for electrically large scattering objects to date. The MoM solution of systems of equations numbering in the tens of millions has been reported [22]. This size problem is potentially within the reach of multicore PCs with several hundred GB of memory. However, there are approximate yet accurate prediction methods available that do not have the memory requirements.

The FDTD method can also be formulated rigorously, and does not require the solution of a large system of equations. It does require discretization of the scattering object and surrounding volume. The incident field is introduced into the computational domain and a marching in time process used to solve Maxwell's equations at each grid location at each instant of time. Equivalence principles are employed to find the far scattered fields from the equivalent currents on the computational boundaries. The FDTD method can require long observation times for accurate results. In the process, a Fourier transform is generally required to obtain the frequency domain fields for use in (1).

The approximate high frequency (HF) methods are primarily based on geometrical optics (GO) or physical optics (PO), and their edge diffraction extensions: the

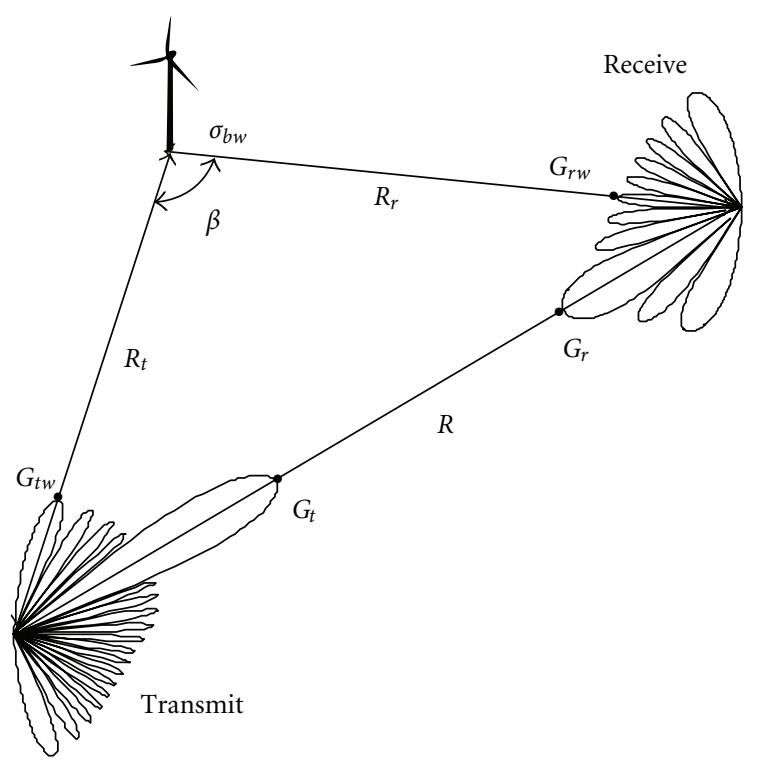

FIgURE 4: Parameters for the bistatic case.

geometrical theory of diffraction (GTD) in the case of GO and the physical theory of diffraction (PTD) for PO [14]. Hybrid solutions methods can include a mix of the two (e.g., the shooting and bouncing ray (SBR) method [23]). The $\mathrm{HF}$ approach also requires surface meshing; however, the primary mesh criterion is that it adequately conforms to the actual surface. A bundle of incident rays is "shot" and traced throughout the model, including transmission through any electrically transparent material, to find reflection points, diffraction points, and shadows (due to blockage). This process can be very time consuming for models that have hundreds of thousands of facets.

There are numerous commercial software packages that can handle the RCS calculations. Several have multiple solvers that can be selected based on the frequency range and object size. High Frequency Structures Simulator (HFSS) by Ansys has transient, frequency domain, and integral equation solvers [24]. The same is true for CST's Microwave Studio [25]. FEKO is also capable of mixed solutions, for example, MoM, PO, GO, and edge diffraction, that can be applied to different portions of the object.

\section{RCS Characteristics of Wind Turbines}

4.1. Introduction. The RCS features of the two generic wind turbine designs shown in Figure 5 are presented in this section. The first is a classic three-blade horizontal axis configuration with approximately a $60 \mathrm{~m}$ tower height and $80 \mathrm{~m}$ blade diameter. The second is a vertical axis helical blade design with a helix diameter of approximately $3 \mathrm{~m}$ and helix height of $3 \mathrm{~m}$. The CAD models were obtained from [26] and scaled to give dimensions in meters. The dimensions are summarized in Table 1.

The software package Lucernhammer (Lucernhammer and $A C A D$ have distribution limitations. They are available only to U.S. Government agencies and contractors.) [27] was used to perform the RCS calculations. Lucernhammer 

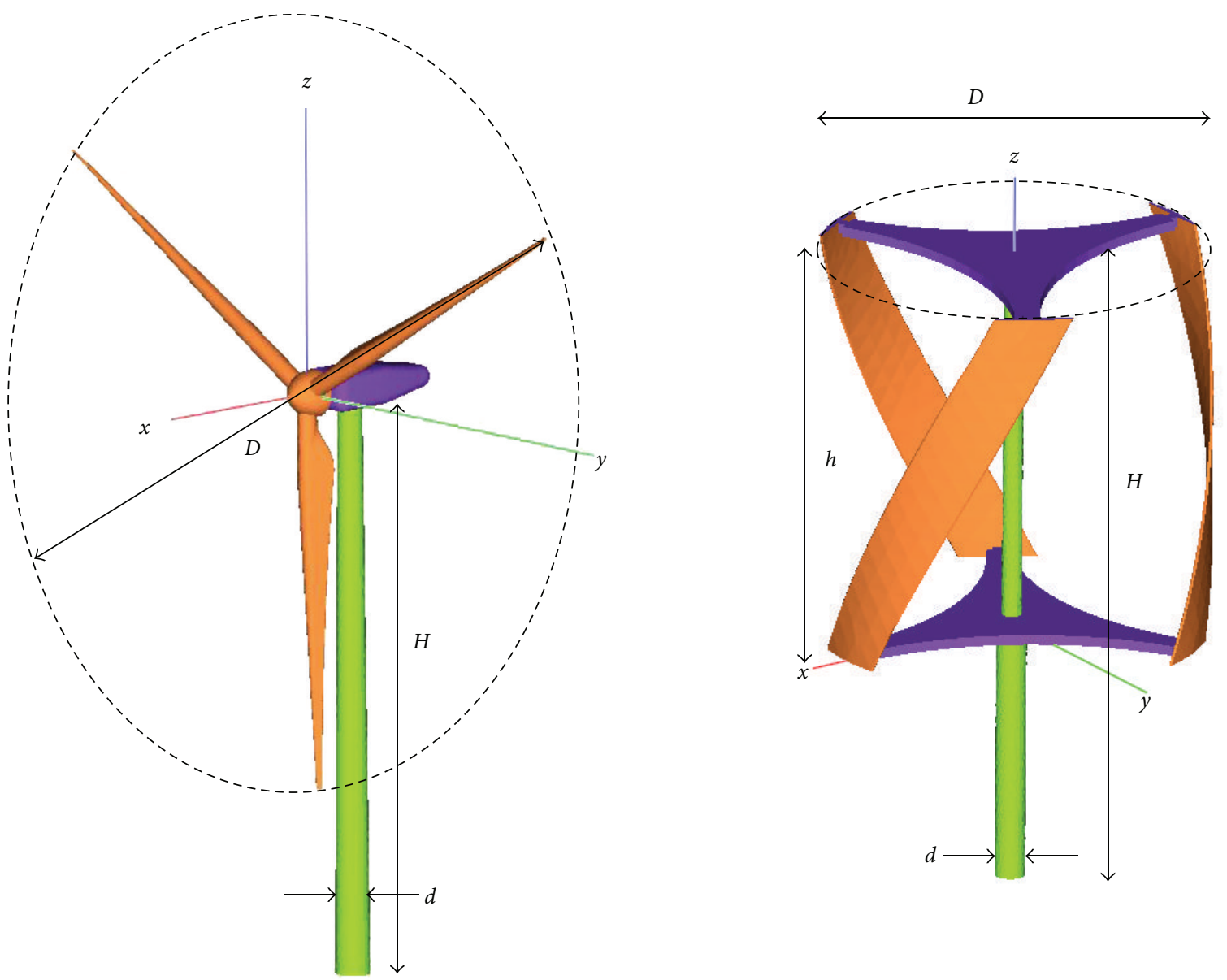

FIGURE 5: Wind turbine models with major dimensions indicated. Various materials are denoted by color.

TABLE 1: Summary of wind turbine dimensions.

\begin{tabular}{|c|c|}
\hline $\begin{array}{l}\text { Three-blade horizontal } \\
\text { axis wind turbine }\end{array}$ & $\begin{array}{l}\text { Vertical axis } \\
\text { helical wind turbine }\end{array}$ \\
\hline Blade diameter, $D=80 \mathrm{~m}$ & $\begin{array}{l}\text { Helix diameter, } D=3 \mathrm{~m} \text {, height, } \\
h=3 \mathrm{~m}\end{array}$ \\
\hline Tower height, $H=60 \mathrm{~m}$ & Tower height, $H=5 \mathrm{~m}$ \\
\hline $\begin{array}{l}\text { Tower diameter at base, } \\
d=3.5 \mathrm{~m}\end{array}$ & $\begin{array}{l}\text { Tower diameter at base, } \\
d=0.5 \mathrm{~m}\end{array}$ \\
\hline $\begin{array}{l}\text { Blade width (widest part), } \\
w=3.6 \mathrm{~m}\end{array}$ & Blade width, $w=0.614 \mathrm{~m}$ \\
\hline
\end{tabular}

employs high frequency methods with ray tracing to compute the RCS of complex shapes. The computer-aided design (CAD) software $A C A D$ was used to generate the surface mesh [28]. Both coarse and fine meshes were used in the calculation of RCS.

The four frequencies considered are representative of wireless, cellular, and radar bands: $400 \mathrm{MHz}, 900 \mathrm{MHz}$, $2400 \mathrm{MHz}$, and $5 \mathrm{GHz}$. No edge diffraction was considered in computing the RCS. Both bistatic and monostatic results are shown, using the coordinate system defined in Figure 2. The $z$-axis points up and the $x-y$ plane is the ground. The elevation angle $\mathrm{EL}=\theta-90^{\circ}$ is measured from the ground.
The azimuth angle is a compass angle that is opposite to $\phi$ $(\mathrm{AZ}=-\phi)$. In all cases only the horizontal plane patterns are shown $\left(\mathrm{EL}=0^{\circ}, \theta=90^{\circ}\right)$, which would be the case when the transmitter, receiver, and wind turbine are at ground level.

RCS data was obtained for models with all surfaces perfect electrically conducting (PEC) and compared to models that had fiberglass blades. The maximum number of ray bounces was set to 5. The non-PEC case is only approximate because Lucernhammer does not trace rays transmitted through the fiberglass blades. This contribution should be negligible though, because the reflection loss at the air-fiberglass boundary is approximately $8 \mathrm{~dB}$ for a fiberglass relative permittivity of $\varepsilon_{r} \approx 5$ [29]. In addition, there is attenuation of the transmitted wave as it propagates through the blade (fiberglass has an electric loss tangent, $\tan \delta \approx$ 0.002 [29]) and an additional reflection loss at the exit surface.

4.2. Horizontal Axis Wind Turbine RCS. Bistatic patterns for the horizontal axis wind turbine are shown in Figures 6 to 9. The blade orientation is such that one blade is vertical and down, as shown in Figure 5 (this is referred to as the $0^{\circ}$ rotation state). Front incidence is $\phi_{i}=0^{\circ}$; side incidence is $\phi_{i}=90^{\circ}$. There is a clear sidelobe structure at the lower two frequencies that arises from the cylindrical tower shape. 

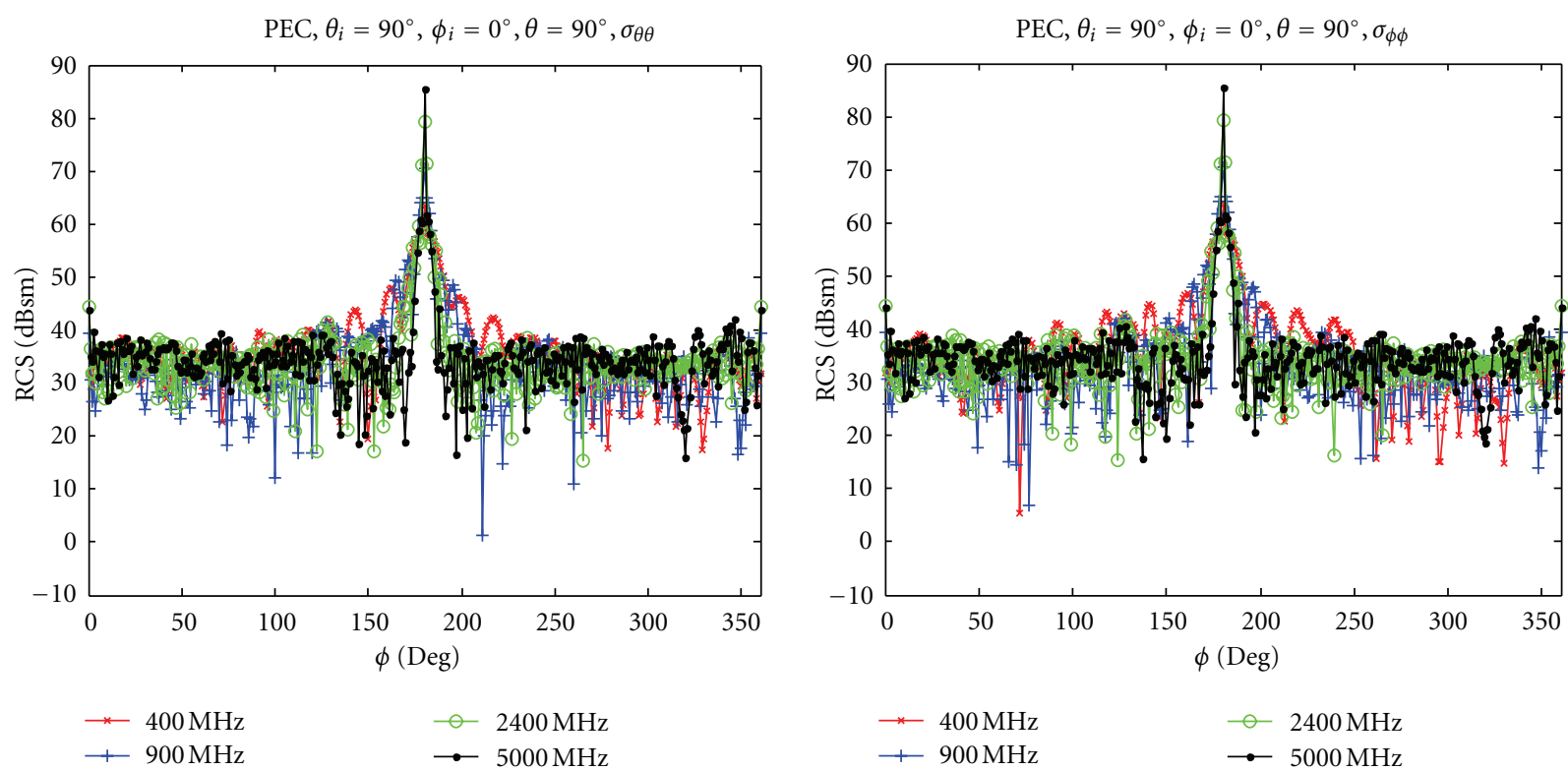

FIgURE 6: Azimuth bistatic copolarized RCS of the horizontal axis wind turbine with all metal surfaces for an incident wave from the front.
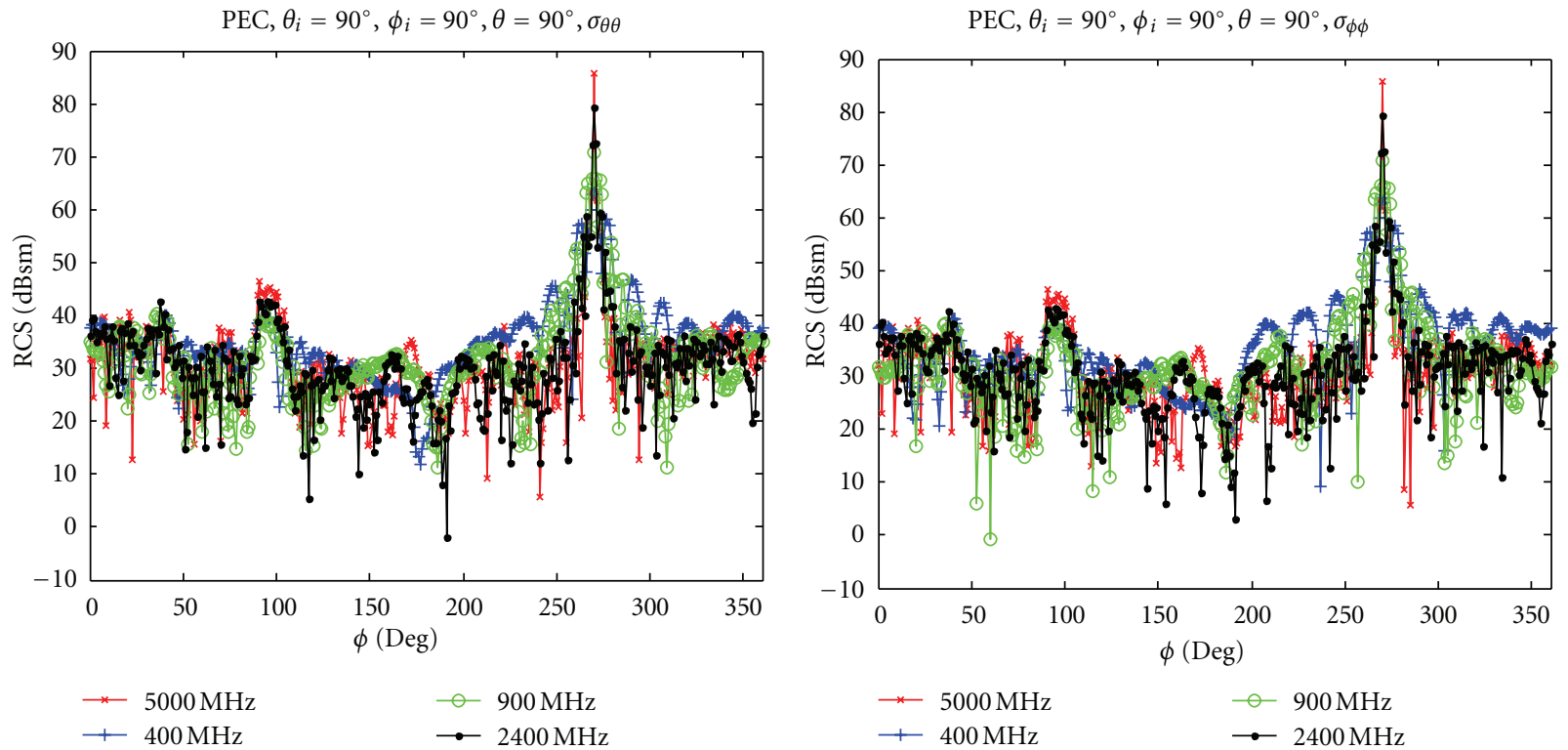

FIgURE 7: Azimuth bistatic copolarized RCS of the horizontal axis wind turbine with all metal surfaces for an incident wave from the side.

What is evident in all of the bistatic plots is the large forward scattering lobe at the observation angle $\phi=\phi_{i}+180^{\circ}$. This occurs at $180^{\circ}$ when incidence is from the front, and at $270^{\circ}$ when incidence is from the side. The forward scatter lobe increases with frequency and is orders of magnitude larger than the backscatter $\left(\phi=\phi_{i}\right)$. This feature is one of the appealing advantages that bistatic radar has over monostatic radar with regard to detecting low RCS (stealthy) targets [18]. The large lobe at $90^{\circ}$ for side incidence is due to specular backscatter from the side of the nacelle.

Figures 6 and 7 are for all metal surfaces, whereas Figures 8 and 9 are for fiberglass blades. Generally wires strands or a wire mesh might be imbedded in the structure for grounding and lightening protection, so the conductor approximation is good at low frequencies. The individual RCS contributions are shown in Figure 10; the bistatic RCS for the nacelle, blades, and tower were computed as if each were isolated in free space. The effect of different blade materials is not all that noticeable. The dominant contribution to the forward scattering is from the tower, which is PEC in all cases.

The copolarized components of the azimuth monostatic RCS for the four frequencies are shown in Figure 11. Lobes occur at all frequencies at 90 and 270 due to the large flat sides of the nacelle. Fluctuations occur with angle as the scattering from the tower, blades, and nacelle adds and cancel with each other. As the frequency is increased, the phase 

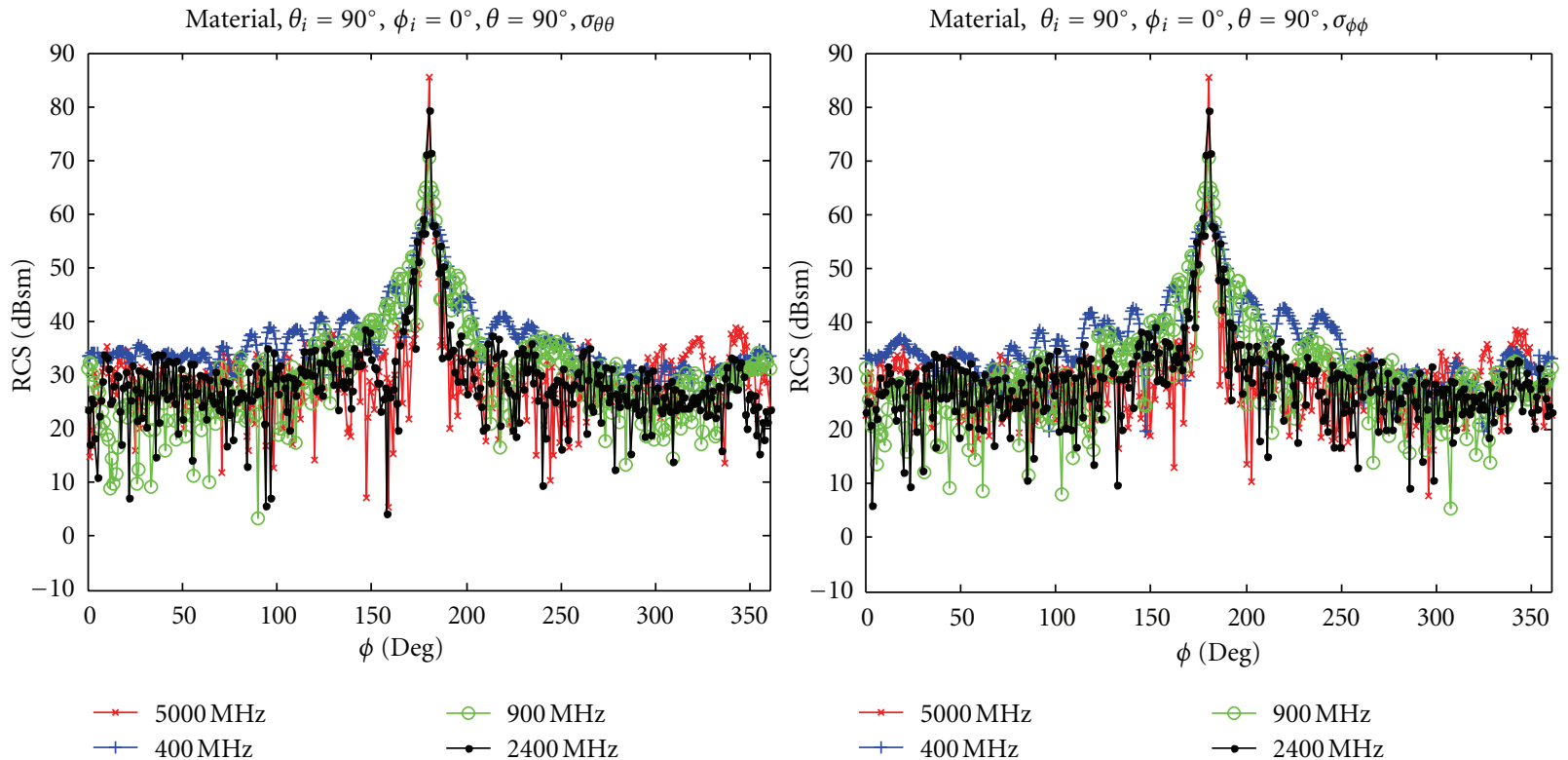

FIgURE 8: Azimuth bistatic copolarized RCS of the horizontal axis wind turbine with fiberglass blades for an incident wave from the front.
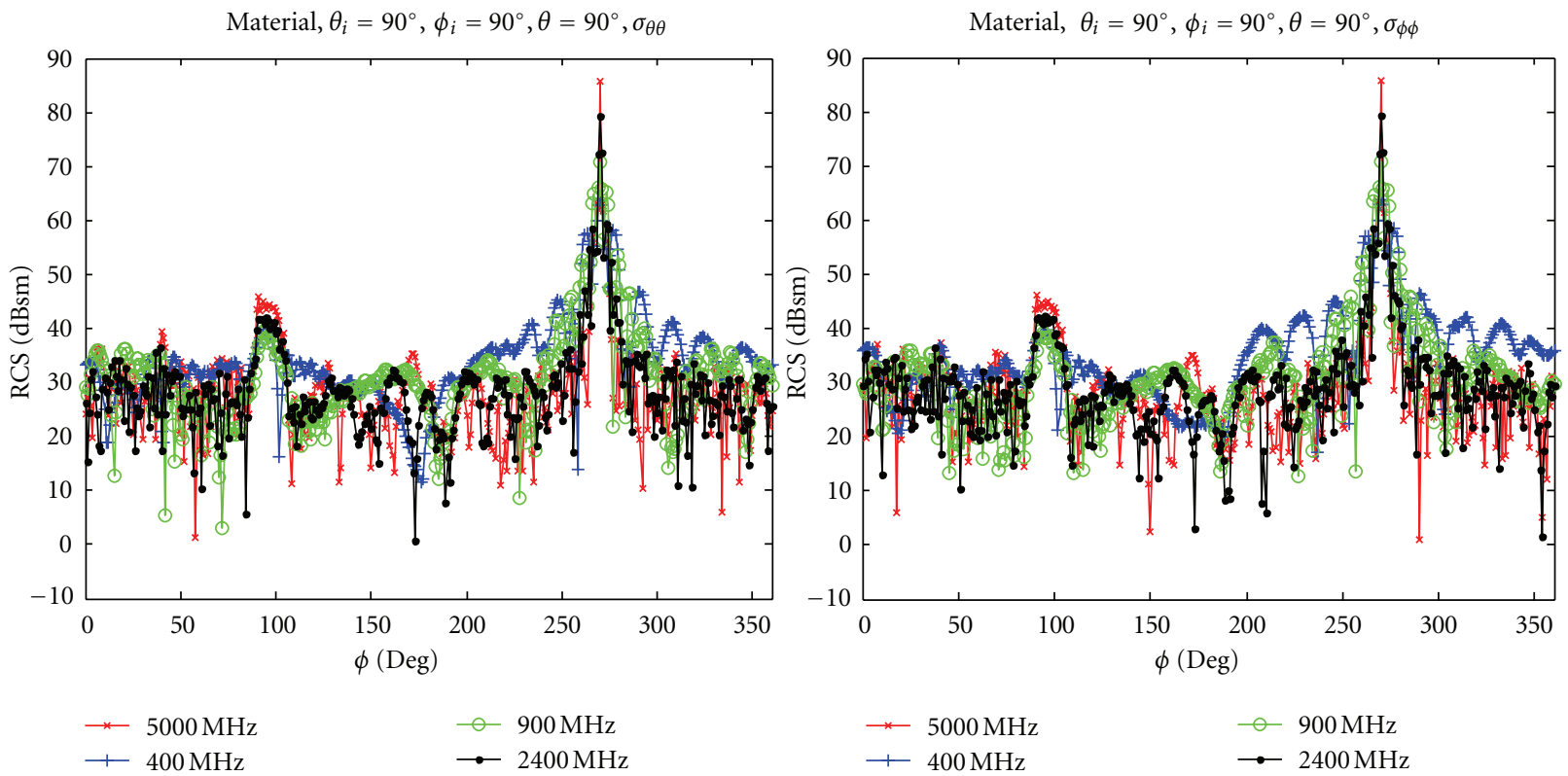

FIGURE 9: Azimuth bistatic copolarized RCS of the horizontal axis wind turbine with fiberglass blades for an incident wave from the side.

differences change more rapidly due to the distances being longer in terms of wavelength. Hence the RCS fluctuates faster with angle.

The relatively high monostatic RCS in the range of 100 to 200 degrees at $400 \mathrm{MHz}$ is due to the tower. The tower sides have a slight tilt back ( 0.6 degrees) because the diameter at the base is larger than at the top. A wave incident at 0 degrees elevation is reflected upward such that an observer at 0 degrees elevation is in the peak of the first sidelobe of the bistatic pattern. At $900 \mathrm{MHz}$ the sidelobes are narrower and an observer at 0 degrees elevation is in a null between two bistatic sidelobes. This is the reason for the large change in RCS between $400 \mathrm{MHz}$ and the higher frequencies.
The periodic oscillations in the RCS in this same region at $400 \mathrm{MHz}$ are due to Bragg scattering from the vertical blade and tower. Figure 12 shows a top view of the relationship between the tower and vertical blade. The round trip phase difference between the blade and tower is a multiple of $2 \pi$ when the condition

$$
2\left(\frac{2 \pi}{\lambda}\right) \ell \sin \left(\phi_{m}\right)=2 m \pi, \quad m=0, \pm 1, \pm 2, \ldots
$$

is satisfied [14], where $\ell$ is approximately $4 \mathrm{~m}$. Equation (10) gives a spacing of about 4 degrees between lobes near broadside (90 degrees) which agrees with the plot in Figure 11. Since the Bragg effect is a "point scatterer" 

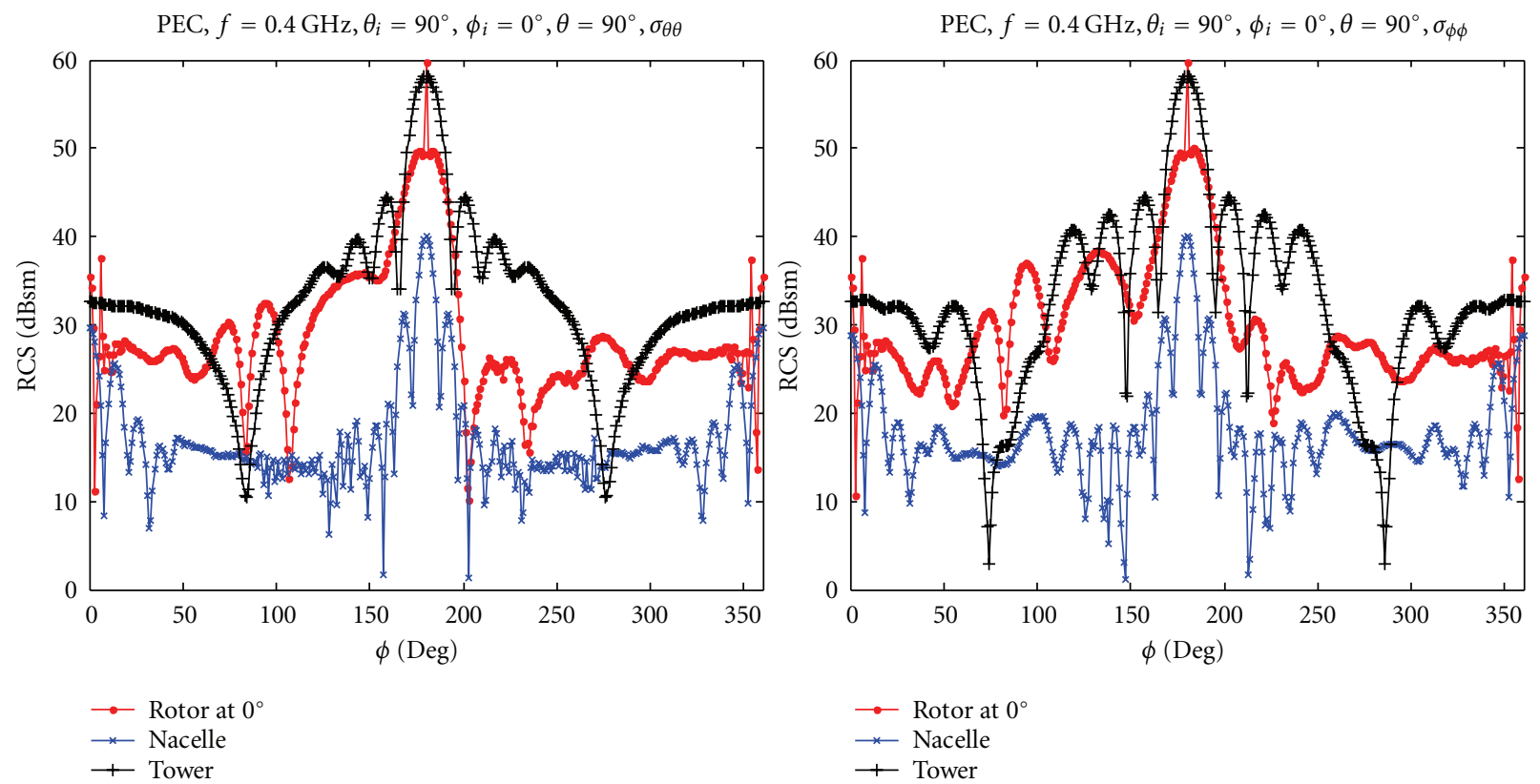

FIGURE 10: Azimuth bistatic copolarized individual RCS contributions of the horizontal axis wind turbine blades, nacelle, and tower for an incident wave from the front at $400 \mathrm{MHz}$.
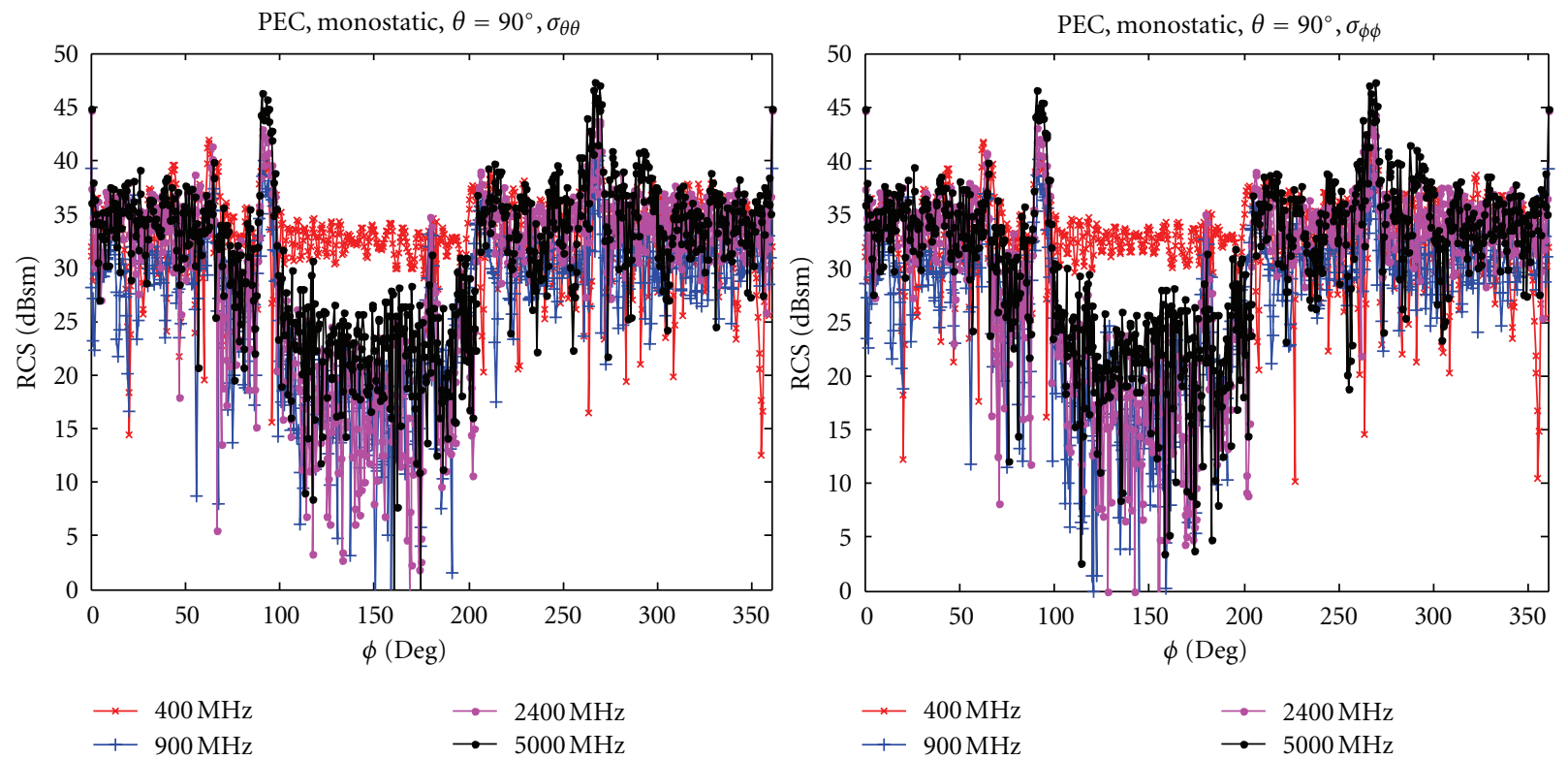

FIGURE 11: Azimuth monostatic copolarized RCS of the horizontal axis wind turbine with all metal surfaces at four frequencies.

phenomenon, it is not as pronounced at higher frequencies, where the surfaces have a larger radius of curvature in terms of wavelength.

The bistatic RCS as a function of rotor position is summarized in Figures 13 and 14, where the RCS for rotor angles from 0 to 120 degrees is plotted in 10 degree steps for the frequency of $400 \mathrm{MHz}$. For the purpose of RCS calculation, the blade rotation is clockwise as viewed from the front. The collected curves are shown on a single figure to illustrate the range of RCS values and highlight the angular regions with the greatest variation. There is no significant change in the regions of the patterns at the higher RCS levels $(>40 \mathrm{~dB})$, but regions with lower RCS can fluctuate 10 to $20 \mathrm{~dB}$.

4.3. Vertical Axis Wind Turbine RCS. Vertical axis wind turbines have been around since the early 1900s. Most have a helical blade geometry, and due to their compact size, they have been proposed as urban rooftop energy solutions [30]. In this section the monostatic and bistatic patterns are presented for the same four frequencies as for the horizontal axis design. The model is shown in Figure 3 with the blades 


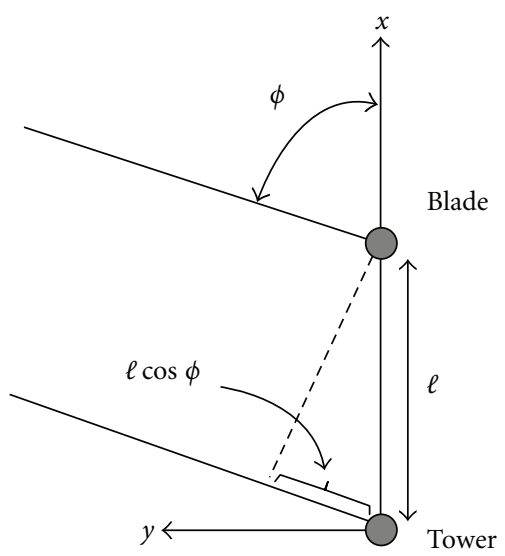

Figure 12: Illustration (top view) of Bragg scattering between the tower and a vertical blade.
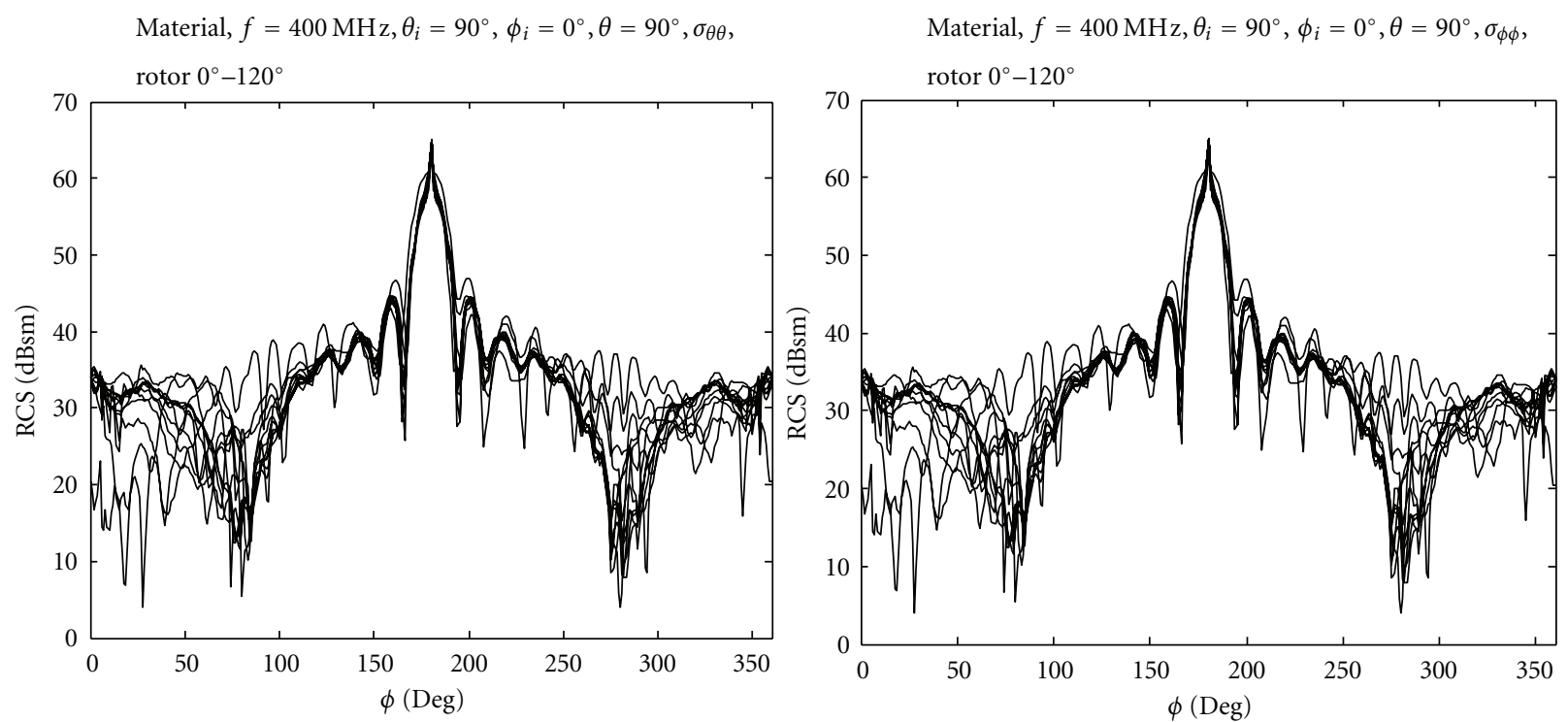

FIgURE 13: Azimuth bistatic copolarized RCS for a collection of blade angles from 0 to 120 degrees in 10 degree increments, front incidence (400 MHz, fiberglass blades).

in the 0 degree position (one blade's bottom centered on the $x$ axis). Front incidence is $\phi_{i}=0^{\circ}$; side incidence is $\phi_{i}=$ $90^{\circ}$. The forward scattered peaks in the bistatic patterns in Figures 15 and 16 are evident at all frequencies. At the lower frequencies, $400 \mathrm{MHz}$ in particular, the sidelobe structure of the tower is visible. The tower diameter is such that it is in the resonance scattering region at $400 \mathrm{MHz}$ [15]. At $5 \mathrm{GHz}$ the multiple narrow lobes are due to Bragg scattering from the blades.

4.4. Computational Issues and Convergence. With a triangular mesh, a large flat rectangular surface can be modeled accurately by as few as two triangles. However, if a large surface is part of a complex target with other scattering elements, and many rays are "shot" for the ray tracing (typically 10 per wavelength), then the larger surface needs to be segmented into smaller surfaces so that blockage and multiple reflections can be accurately determined. More segmentation increases the accuracy but also increases the run time. In the case of a flat surface there is a relationship between segmentation and mesh size. If a large plate is meshed with small triangles, then less segmentation is required.

The RCS plots in Figure 17 give an example of how the segmentation value (i.e., segments per triangle edge) affects the RCS. With adequate segmentation the perturbation of RCS is relatively small, and thus tends to be an issue for only cross-polarized RCS components.

For curved surfaces, the mesh size must be fine enough (i.e., sufficiently small triangle edges) so that a "tight" fitting mesh can be generated. Even so, when a curved surface is approximated by a triangular mesh, facet noise will occur (it is also called facetization error) [31]. In a general sense facet noise can be categorized as a quantization error that arises from representing the smooth continuous surface with discrete facets. 

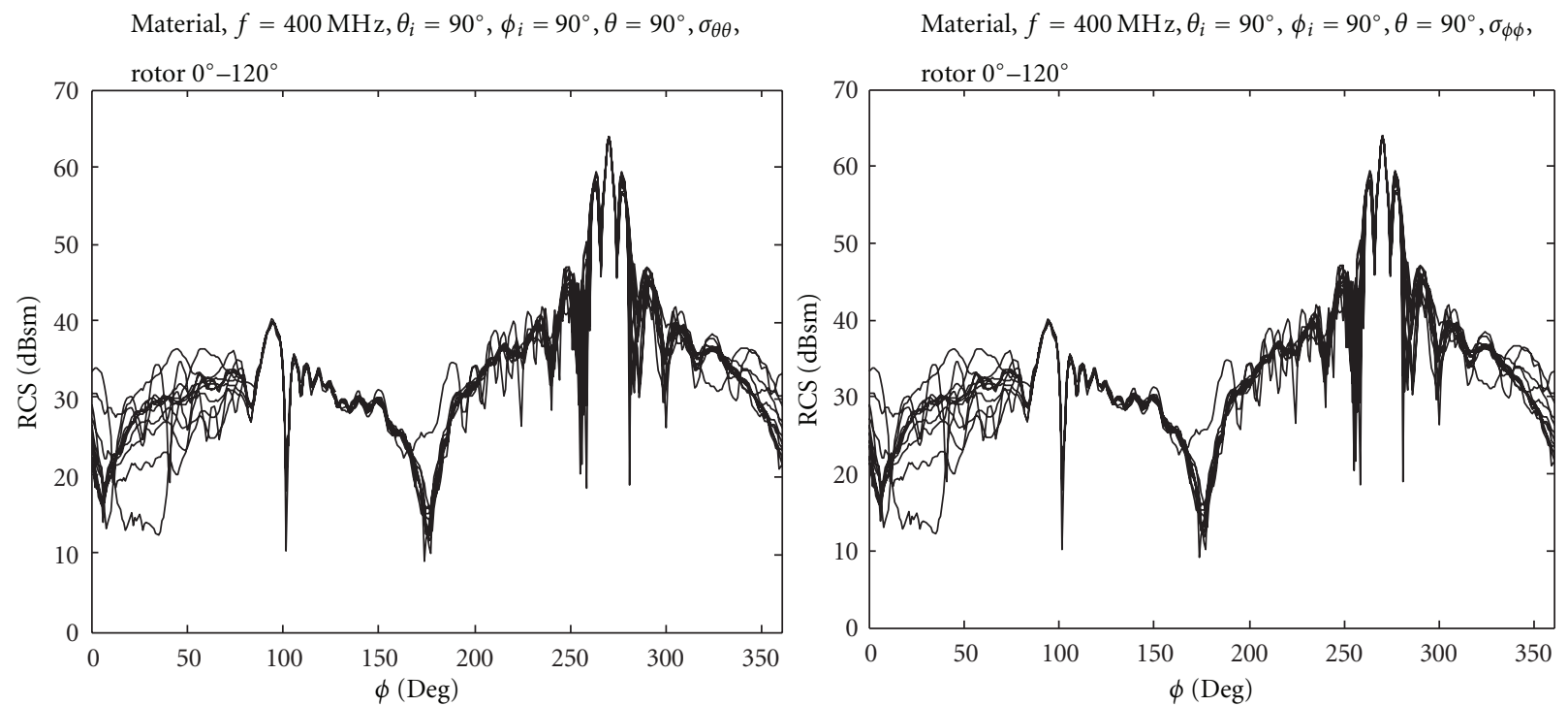

FIGURE 14: Azimuth bistatic copolarized RCS for a collection of blade angles from 0 to 120 degrees in 10 degree increments, side incidence (400 MHz, fiberglass blades).
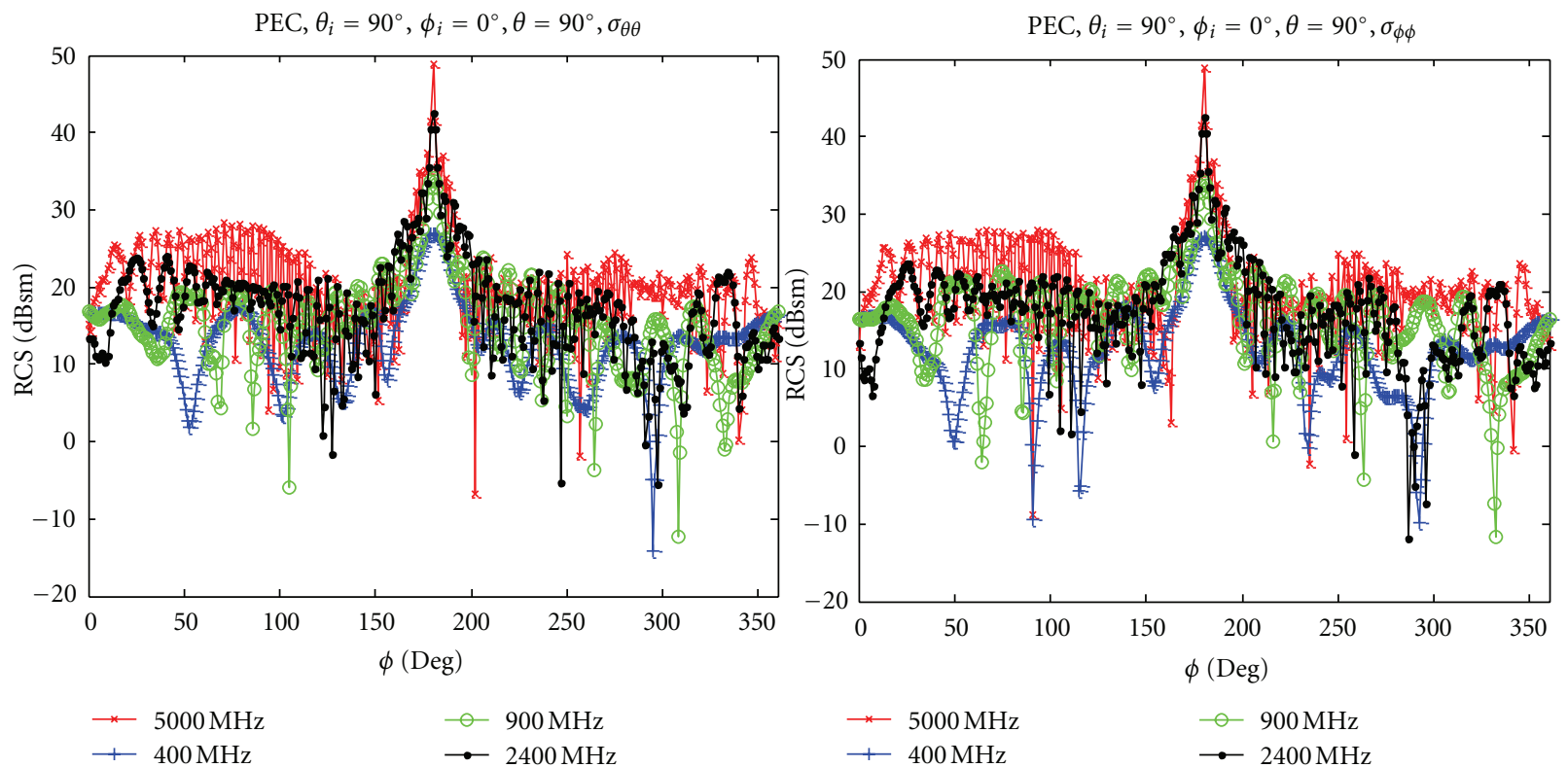

FIgURE 15: Bistatic copolarized RCS of the helical wind turbine, all metal surfaces, front incidence.

To observe the effects of facetization error, the monostatic RCS of the tower is computed. Ideally the azimuth monostatic RCS should be constant with angle because the tower is a body of revolution. In Figure 18 are shown three mesh models of the $60 \mathrm{~m}$ high horizontal axis wind turbine's tower. Two are triangular meshes with different densities, and the third is a quadrilateral triangular mesh. A quadrilateral triangular mesh is obtained by first meshing the surface into quadrilaterals and then making triangles by adding the diagonals. Long thin vertical quadrilaterals can be used on the tower because it is essentially a singly curved surface; small segments only need to be used around the circumference of the tower. This results in significantly fewer facets for the tower.
Figure 19 has a comparison of the monostatic RCS for a 90-degree sector at $900 \mathrm{MHz}$ for the three meshes in Figure 18. The coarse mesh results vary by $20 \mathrm{~dB}$, and would generally be considered unacceptable. However, in the forward scattered direction, the peak RCS level of the bistatic RCS at this frequency is about $70 \mathrm{~dB}$, so this level of facet noise may be acceptable. Even the RCS for the fine mesh has variations of approximately $2 \mathrm{~dB}$. The quadrilateral/triangular mesh has the same accuracy as the fine triangular mesh using only $3.7 \%$ of the fine mesh's number of facets. The reduction yields a significant computational savings when calculating the RCS for the entire wind turbine.

In Figure 20 are shown the bistatic patterns of the horizontal axis wind turbine for the three meshes at a frequency 

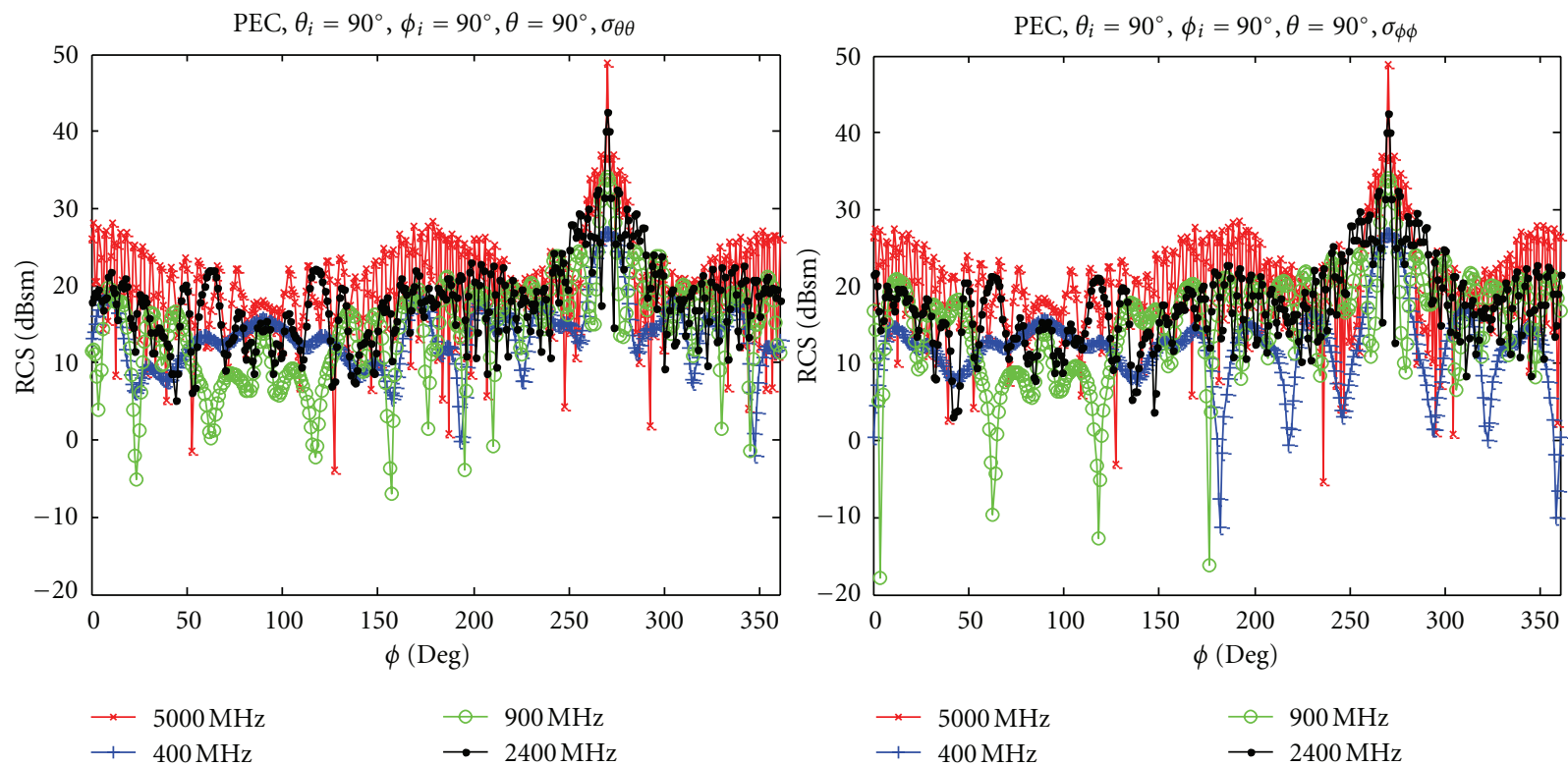

FIGURE 16: Bistatic copolarized RCS of the helical wind turbine, all metal surfaces, side incidence.
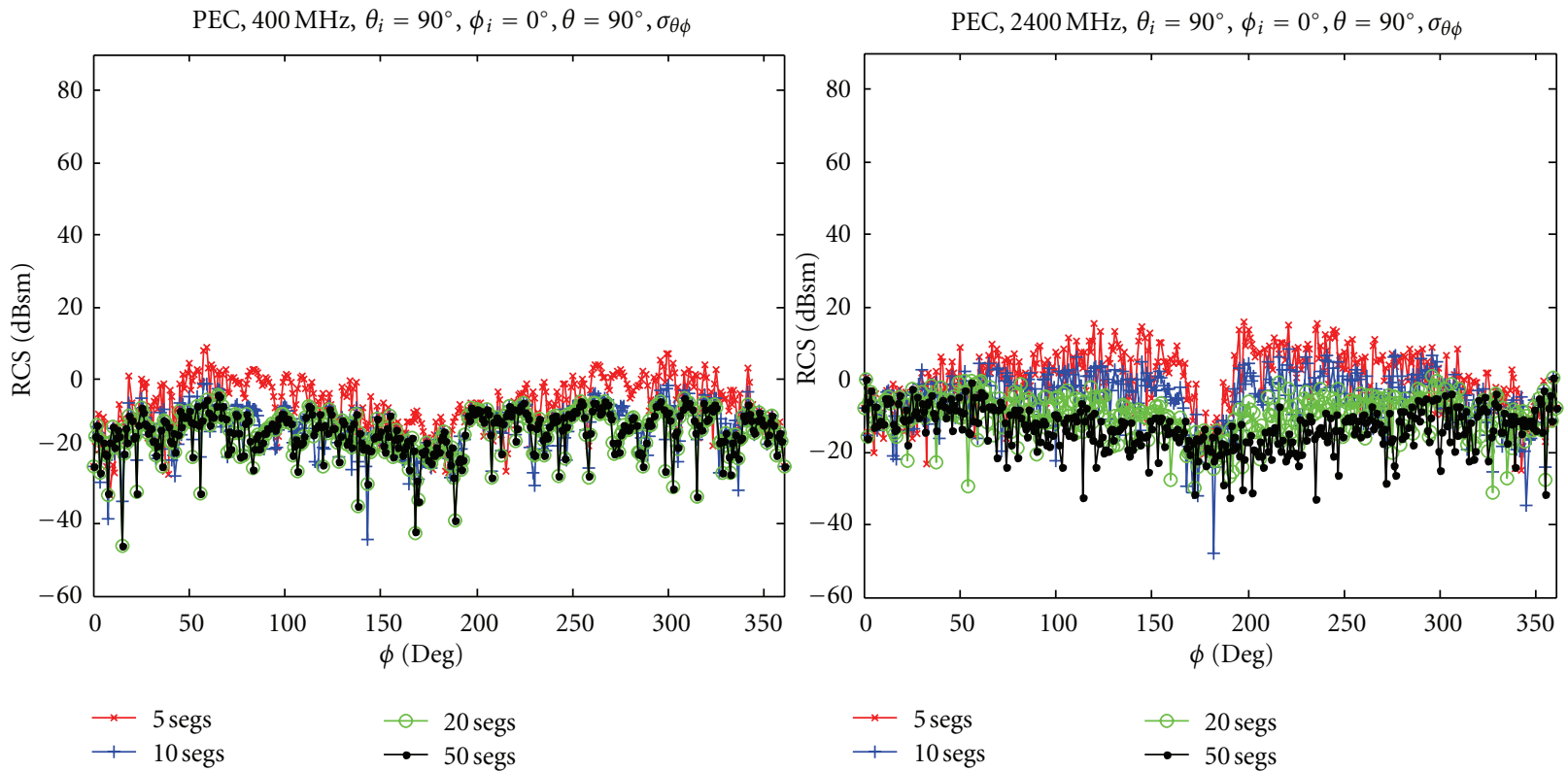

Figure 17: An example of the bistatic azimuth cross-polarized RCS variation due to the segmentation parameter, PEC horizontal axis wind turbine, front incidence.

of $900 \mathrm{MHz}$. It is apparent that the cross-polarized components are more sensitive to facet noise because of the lower values of RCS.

\section{RCS Reduction and Control}

For both the monostatic and bistatic cases, the SCR, given by (5) and (9), can be increased by reducing the wind turbine RCS. Traditionally there are three approaches to reducing RCS: (1) shaping, (2) application of radar absorbing materials, and (3) cancellation techniques $[14,15]$.

Shaping applied to the tower and nacelle could be somewhat effective, but it would have to be done with knowledge of the transmitter and receiver directions. Although it could reduce the RCS in some desired monostatic or bistatic directions, it would likely increase it in others. Shaping of wind turbine structures to reduce RCS has been investigated in [13].

Cancellation techniques involve the introduction of secondary scatterers to cancel the wind turbine's RCS (i.e., so as to induce destructive interference). It requires phase coherence between the primary (wind turbine) and secondary scattering components. This would be very difficult to achieve, especially at the higher frequencies, and it is only effective at limited frequencies and angles. Furthermore, 

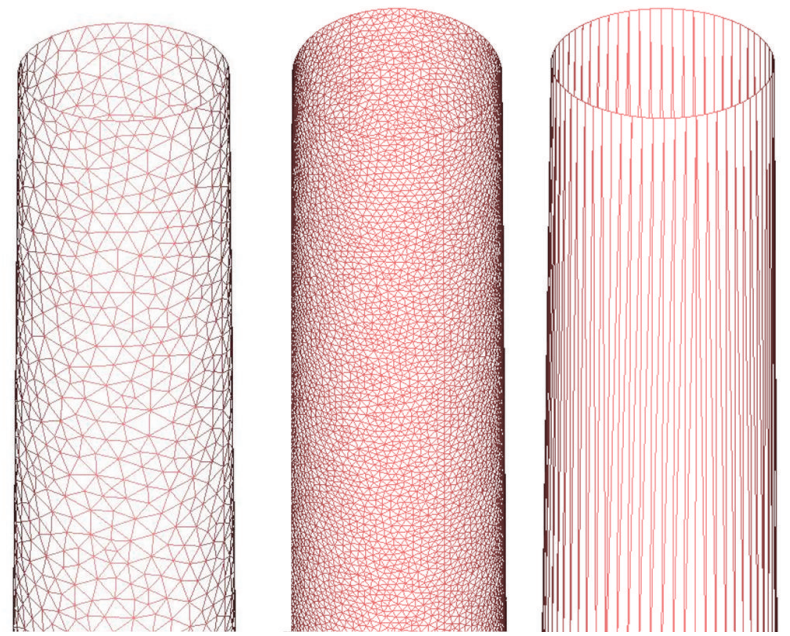

FIGURE 18: Illustration of mesh types for the tower (left to right): coarse triangular (19,994 facets), fine triangular (125240 facets), and quadrilateral triangular meshes (4608 facets).

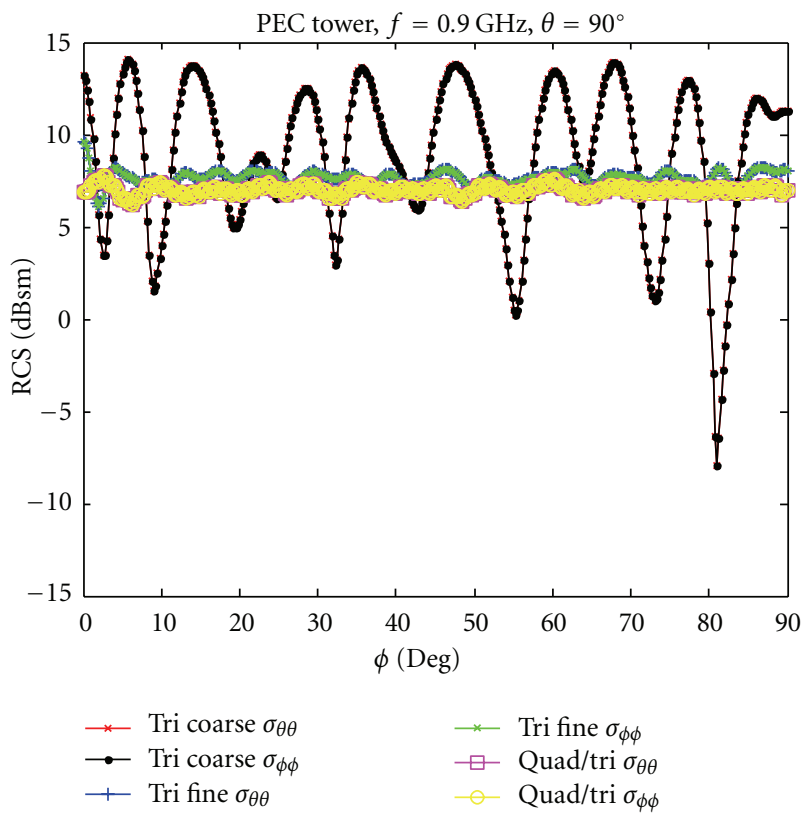

FIGURE 19: Azimuth copolarized monostatic RCS of the PEC tower for three meshes: coarse triangular (19994 facets), fine triangular (125240 facets), and quadrilateral triangular (4608 facets).

the secondary scatterer would have to be very large in order to cancel the large wind turbine RCS.

The most promising approach is the application of radar absorbing material (RAM). The material would have to be lightweight, thin, durable, inexpensive, and provide sufficient RCS reduction to make it economically viable. Most commercial RAM materials give a specular RCS reduction in the range of 15 to $20 \mathrm{~dB}$ (see, e.g., Emerson and Cuming Eccosorb FGM [32]); however, it varies widely with frequency and angle of incidence. A RAM coating might make sense if the wind turbine was at a fixed location from a facility, such as an airport radar. However, the bistatic RCS is so large that the aerodynamic degradation of the blades and cost of adding RAM would not generally be merited.

\section{Summary and Conclusions}

The equations for the signal-to-clutter ratio were presented for the monostatic and bistatic cases. The equations show that the SCR can be increased by

(i) reducing the wind turbine RCS, or operating in a direction where the RCS is low,

(ii) reducing the antenna sidelobe levels so that the gain is lower in the direction of the wind turbine, or

(iii) in the case of radar, operating in a condition where the target range is much closer than the wind turbine.

The high-frequency computational method physical optics was used to predict the RCS. It has the advantage of 

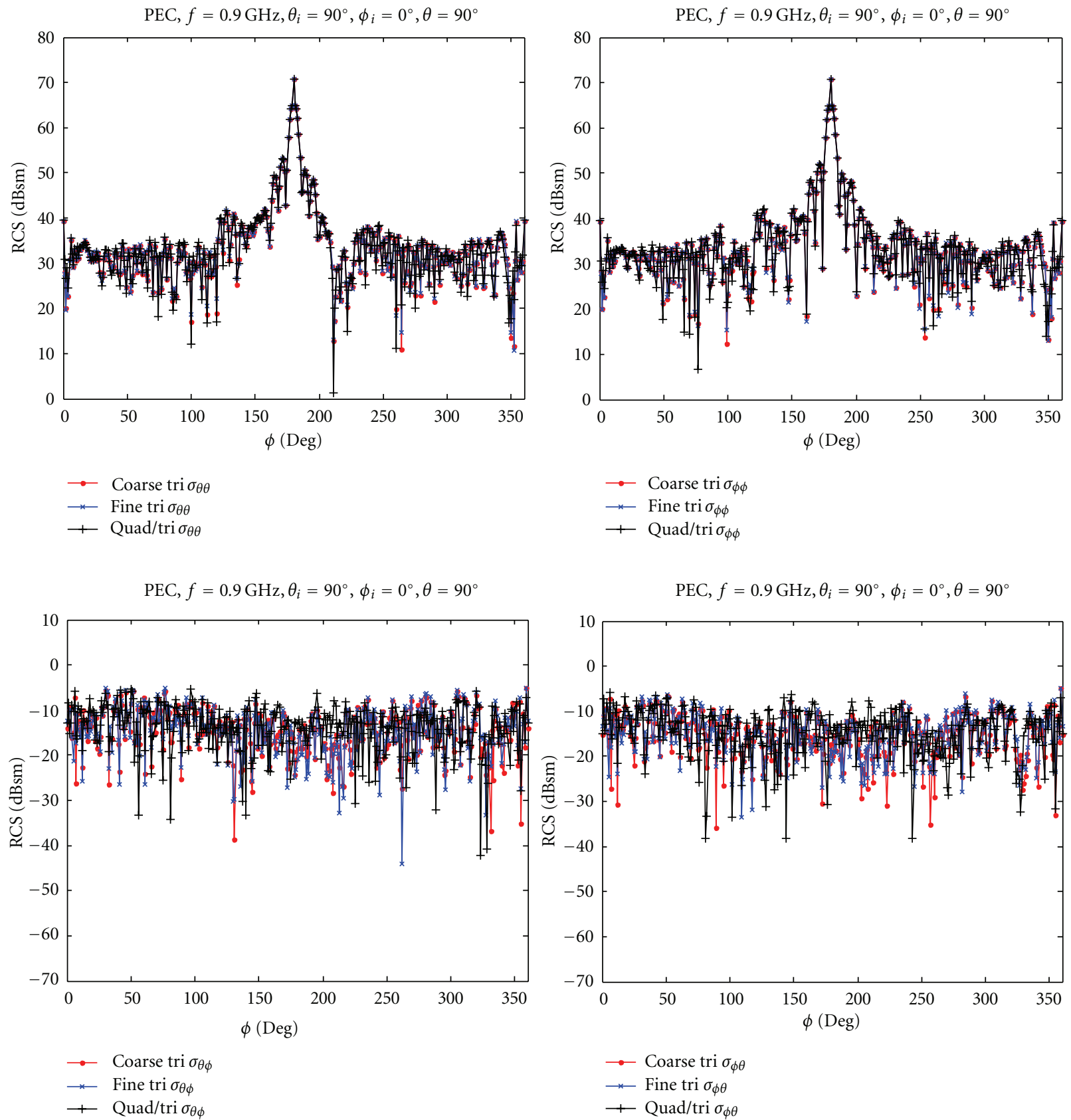

Figure 20: Comparison of the four components of bistatic RCS for the three meshes at $900 \mathrm{MHz}$, all PEC, front incidence.

not requiring the solution of a large number of simultaneous equations. The computational convergence issues related to surface meshing, number of bounces, and segmentation of the edges were discussed. The quadrilateral/triangular mesh on the tower provides the same accuracy as a fine triangular mesh with only $3.7 \%$ of the fine triangular mesh's number of facets. This reduction yields a significant computational time savings when calculating the RCS for the entire wind turbine.

RCS patterns were presented for two wind turbine configurations: a three-blade horizontal axis design and a helical vertical axis design. The behavior of the RCS is a complicated function of angle, frequency, and rotor position. Both wind turbine configurations have relatively high forward scatter RCS that increases with frequency. For the horizontal axis models with tall PEC towers, the RCS is dominated by the tower scattering. The finer structure of the patterns varies with rotor position, and Bragg scattering can be observed in some situations. The reduction in RCS by using nonconducting blade materials was not significant.

Lastly, the possibility of reducing the radar cross section by the application of RAM was discussed. In most situations, the aerodynamic degradation and cost would not merit the use of RAM given the relatively small reduction in RCS that it would provide. An exception might be when wind farms operate in the vicinity of radar systems, in which case shaping and RAM can be used effectively. 


\section{References}

[1] "Wind power by country," Wikipedia, October 2012, http://en .wikipedia.org/wiki/Wind_power_by_country.

[2] Global Wind Energy Council, "Global wind capacity increases by $22 \%$ in 2010 - Asia leads growth," 2011, http://www.gwec .net/.

[3] C. Lute and W. Wieserman, "ASR-11 radar performance assessment over a wind turbine farm," in Proceedings of IEEE Radar Conference (RADAR '11), pp. 226-230, 2011.

[4] L. S. Rashid and A. K. Brown, "RCS and radar propagation near offshore wind farms," in Proceedings of IEEE Antennas and Propagation Society International Symposium, pp. 4605-4608, Honolulu, Hawaii, USA, June 2007.

[5] D. Sozen and M. Kartal, "Scatter and doppler effect of wind power plants to land radars," in Proceedings of the 14th International Conference on Modeling and Simulation, pp. 453458, IEEE Computer Society, 2012.

[6] B. M. Kent, K. C. Hill, A. Buterbaugh et al., "Dynamic radar cross section and radar doppler measurements of commercial general electric windmill power turbines part 1: predicted and measured radar signatures," IEEE Antennas and Propagation Magazine, vol. 50, no. 2, pp. 211-219, 2008.

[7] J. C. G. Matthews, C. Sarno, and R. Herring, "Interaction between radar systems and wind farms," in Proceedings of Loughborough Antennas and Propagation Conference (LAPC '08), pp. 461-464, Loughborough, UK, March 2008.

[8] F. Kong, Y. Zhang, R. Palmer, and Y. Bai, "Wind turbine radar signature characterization by laboratory measurements," in Proceedings of IEEE Radar Conference (RADAR '11), pp. 162166, Kansas City, Mo, USA, May 2011.

[9] E. van Lil, J. W. de Bleser, and A. van de Capelle, "Computations of the effects of wind turbines in the close near field of RF installations," in Proceedings of the 5th European Conference on Antennas and Propagation (EUCAP '11), pp. 1362-1366, Rome, Italy, April 2011.

[10] I. Angulo, D. de la Vega et al., "Experimental results on wind turbines impact to terrestrial DTV reception in the UHF band," in Proceedings of the 59th Annual IEEE Broadcast Symposium, Alexandria, Va, USA, October 2009.

[11] L. Rashid and A. Brown, "Radar cross section analysis of wind turbine blades with radar absorbing material," in Proceedings of the 8th European Radar Conference, pp. 97-100, October 2011.

[12] J. Lord, J. Matthews, and J. Pinto, "RCS predictions for stealthy wind turbines," in Proceedings of Conference on Antennas and Propagation (EuCAP '06), pp. 6-10, Nice, France, 2006.

[13] J. Pinto, J. Matthews, and G. C. Sarno, "Stealth technology for wind turbines," IET Radar Sonar Navig, vol. 4, no. 1, pp. 126$133,2000$.

[14] E. Knott, J. Shaffer, and M. Tuley, Radar Cross Section, Artech House, London, UK, 2nd edition, 1993.

[15] D. Jenn, Radar and Laser Cross Section, AIAA Education Series, 2nd edition, 2005.

[16] M. I. Skolnik, Introduction to Radar Systems, McGraw-Hill, New York, NY, USA, 3rd edition, 2001.

[17] W. Stutzman and G. Thiele, Antenna Theory and Design, John Wiley \& Sons, New York, NY, USA, 2nd edition, 1998.

[18] N. Willis, Bistatic Radar, Artech House, London, UK, 2nd edition, 1991.

[19] C. Balanis, Advanced Engineering Electromagnetics, McGraw Hill, New York, NY, USA, 2012.
[20] K. Kunz and R. Luebbers, The Finite Difference Time Domain Method for Electromagnetics, CRC Press, Boca Raton, Fla, USA, 1993.

[21] J. Volakis, A. Chatterjee, and L. Kempel, Finite Elements Method for Electromagnetics, IEEE Press, Piscataway, NJ, USA, 1998.

[22] J. M. Taboada, L. Landesa, F. Obelleiro et al., "High scalability FMM-FFT electromagnetic solver for supercomputer systems," IEEE Antennas and Propagation Magazine, vol. 51, no. 6, pp. 20-28, 2009.

[23] H. Ling, R. C. Chou, and S. W. Lee, "Shooting and bouncing rays: calculating the RCS of an arbitrarily shaped cavity," IEEE Transactions on Antennas and Propagation, vol. 37, no. 2, pp. 194-205, 1989.

[24] ANSYS HFSS, October 2012, http://www.ansys.com/.

[25] CST MWS, October 2012, http://www.cst.com/Content/ Products/MWS/Overview.aspx.

[26] 3D Content Central, CAD model, October 2012, http://www .3dcontentcentral.com/Search.aspx?arg=wind\%20turbine.

[27] Tripoint Industries, October 2012, http://lucernhammer.tripointindustries.com/.

[28] U.S. Air Force Research Laboratory Supercomputing Resource Center, October 2012, http://www.afrl.hpc.mil/software/info/ acad/\#desc.

[29] "Fiberglass Compositions and Their Comparative Properties," Ceramic Industry, http://www.ceramicindustry.com/ext/resources/CI/Protected/Files/PDF/fiberglass-compositions.pdf.

[30] B. C. Howard, "Helix Collapse Fails to Crush Hope for Vertical Wind Turbines," National Geographic Daily News, October 2012, http://news.nationalgeographic.com/news/energy/ 2012/08/120820-helix-wind-collapse/.

[31] E. M. Miller, D. J. Andersh, and A. J. Terzuoli, "Effect of target model facetization on RCS predictions," in Proceedings of the IEEE International Symposium Digest of Antennas and Propagation, pp. 1404-1407, July 1993.

[32] Emerson and Cuming, October 2012, http://www.eccosorb .com/products-eccosorb-fgm.htm. 

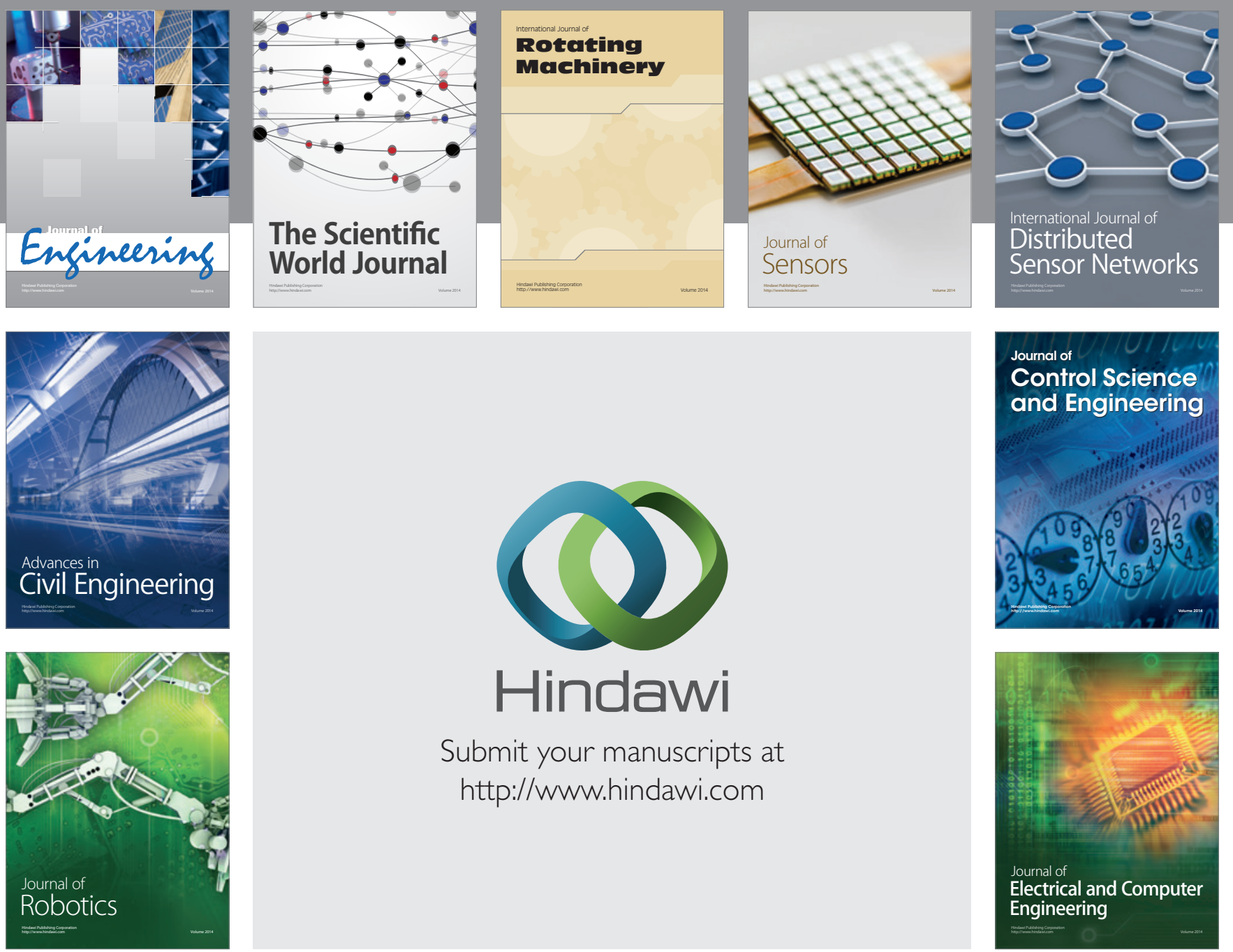

Submit your manuscripts at

http://www.hindawi.com
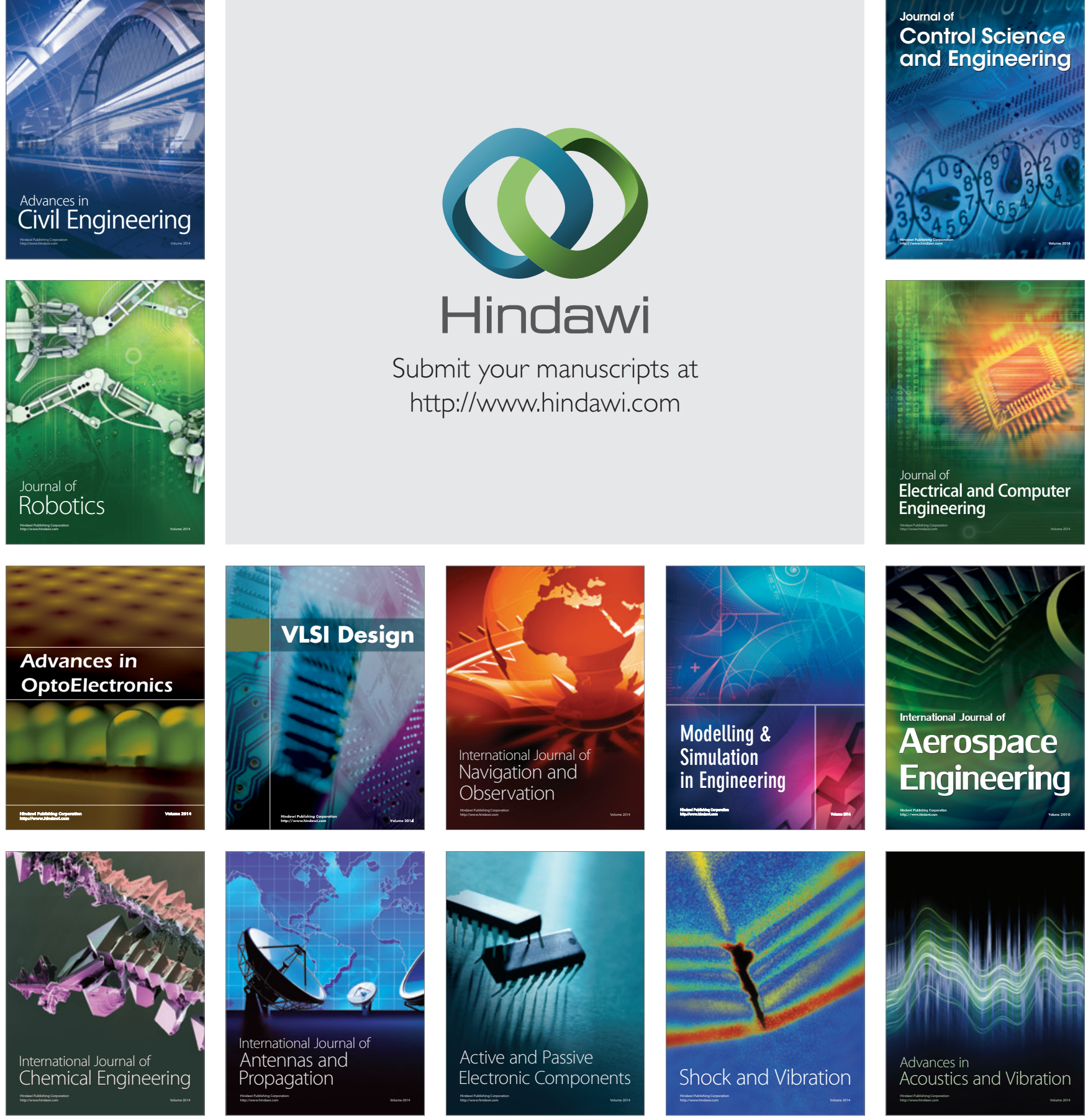